PROCEEDINGS OF THE

AMERICAN MATHEMATICAL SOCIETY

Volume 132, Number 11, Pages 3417-3421

S 0002-9939(04)07416-7

Article electronically published on June 17, 2004

\title{
THIN POSITION AND ESSENTIAL PLANAR SURFACES
}

\author{
YING-QING WU
}

(Communicated by Ronald A. Fintushel)

\begin{abstract}
Abby Thompson proved that if a link $K$ is in thin position but not in bridge position, then the knot complement contains an essential meridional planar surface, and she asked whether some thin level surface must be essential. This note is to give a positive answer to this question, showing that if a link is in thin position but not bridge position, then a thinnest level surface is essential. A theorem of Rieck and Sedgwick follows as a consequence, which says that thin position of a connected sum of small knots comes in the obvious way.
\end{abstract}

The concept of thin position was introduced by David Gabai in [G], and has been used successfully in attacking some very difficult problems; see, for example, G], GL], [ST], and [T1]. In T2 Abby Thompson proved that if a knot $K$ is in thin position but not in bridge position, then some thin level surface can be compressed to produce an essential planar surface in the complement of $K$ with meridional boundary slope; in particular, by a theorem of Culler, Gordon, Luecke and Shalen [CGLS] this implies that the knot is large in the sense that its complement contains some closed essential surfaces. This was further explored by Heath and Kobayashi [HK], who showed that a certain thin level surface of a nonsplit link $L$ in thin position but not bridge position can be compressed to give some natural tangle decomposition of $L$, and the decomposing spheres then give rise to essential meridional planar surfaces in the link complement. In both [T2] and [HK] the essential planar surfaces come from compression of a thin level surface. There are examples in [HK] showing that some essential meridional planar surfaces are not level surfaces of a knot in thin position. This leads to a question raised by Thompson [T3], which asks whether a link $L$ in thin position but not in bridge position has a level surface that is essential. The purpose of this paper is to give a positive solution to this problem.

Theorem 1. If a link $L$ in $S^{3}$ is in thin position but not in bridge position, then a thinnest level surface $Q$ of $L$ is an essential surface in $S^{3}-\operatorname{Int} N(L)$.

We give some definitions. Consider $S^{3}$ as $\mathbb{R}^{3} \cup\{\infty\}$, and let $\rho$ be the height function $\rho: \mathbb{R}^{3} \rightarrow \mathbb{R}$ defined by $\rho(x, y, z)=z$. For each $t \in \mathbb{R}$, let $P(t)=\rho^{-1}(t)$ be the horizontal plane in $\mathbb{R}^{3}$ at height $t$. When $t$ is not a critical level of $\rho$, define $Q(t)$ to be the punctured sphere $(P(t) \cup\{\infty\})-\operatorname{Int} N(L)$, called the level surface at level

Received by the editors February 27, 2003 and, in revised form, June 16, 2003.

2000 Mathematics Subject Classification. Primary 57M25.

Key words and phrases. Thin position, knots and links, essential planar surfaces.

Partially supported by NSF grant \#DMS 0203394.

(C)2004 American Mathematical Society 
$t$, where $N(L)$ is a small regular neighborhood of $L$ intersecting $P$ in meridional disks. If $I$ is an interval on $\mathbb{R}$, denote by $Z_{I}=\mathbb{R}^{2} \times I$ the set of points in $\mathbb{R}^{3}$ whose $z$-coordinate is in $I$.

Let $L$ be a link in $\mathbb{R}^{3}$ such that the restriction of $\rho$ to $L$ is a Morse function, and let $a_{0}, \ldots, a_{n}$ be the critical points of $L$, labeled so that the corresponding critical values $t_{i}=\rho\left(a_{i}\right)$ satisfy $t_{i-1}<t_{i}$ for all $i$. Let $s_{i} \in\left(t_{i-1}, t_{i}\right)$. Thus $P_{i}=P\left(s_{i}\right)$ is a plane between $a_{i-1}$ and $a_{i}$, called a level plane corresponding to the critical point $a_{i-1}$. The width of $P(t)$ (with respect to $L$ ) is defined as $w(P(t))=|P(t) \cap L|$, where $|A|$ denotes the number of elements in $A$. The width of $L$ is $w(L)=\sum_{1}^{n} w\left(P_{i}\right)$. A link $L$ is in thin position if $w(L)$ is minimal up to isotopy of $L$.

A plane $P(t)$ with $t_{i-1}<t<t_{i}$ is called a thin level plane of $L$, and $t$ a thin level, if $a_{i-1}$ is a local maximum and $a_{i}$ a local minimum. Similarly, $t \in\left(t_{i-1}, t_{i}\right)$ is a thick level and $P(t)$ a thick level plane if $a_{i-1}$ is a local minimum and $a_{i}$ a local maximum. A thin level plane $P(t)$ is a thinnest level plane and the corresponding planar surface $Q(t)=(P(t) \cup\{\infty\})-\operatorname{Int} N(L)$ a thinnest level surface if $w(P(t))$ is minimal among all thin planes. The link $L$ is in bridge position if it has no thin level.

Proof of Theorem 1. Let $P$ be a thinnest level plane, and let $Q$ be the corresponding planar surface defined above. Moving $L$ up or down if necessary, we may assume without loss of generality that $P=P(0)$, i.e., it is the $(x, y)$-plane in $\mathbb{R}^{3}$. Our goal is to show that $Q$ is an essential surface in $E(L)$. Recall that a properly embedded compact orientable surface in a 3-manifold $M$ is essential if (i) it is incompressible (in particular it is not a 2-sphere bounding a 3-ball), and (ii) it is not boundary parallel. Since $P$ is a thin level plane, it is easy to show that $Q$ is never a 2 -sphere bounding a 3-ball, and it is not boundary parallel. Therefore, we need only show that it is not a compressible punctured sphere.

Assume to the contrary that $Q$ is compressible, and let $D$ be a compressing disk. Without loss of generality we may assume that $D$ is in the upper half space $\mathbb{R}_{+}^{3}=Z_{[0, \infty)}$. We will show below that either $L$ is not in thin position or $P$ is not a thinnest level plane, which will contradict the assumption and complete the proof of the theorem.

The $(x, z)$-coordinate plane cuts $\mathbb{R}_{+}^{3}$ into $Y_{-}$and $Y_{+}$, where $Y_{-}$is the left half space of $\mathbb{R}_{+}^{3}$, consisting of points in $\mathbb{R}_{+}^{3}$ with negative $y$-coordinate, and $Y_{+}$the right half space of $\mathbb{R}_{+}^{3}$. Let $D^{\prime}$ be the disk on $P$ with $\partial D=\partial D^{\prime}$, and let $B$ be the 3-ball bounded by $D \cup D^{\prime}$. Define $\alpha=L \cap B$ and $\beta=L \cap\left(\mathbb{R}_{+}^{3}-B\right)$. Since $D$ is a compressing disk of $Q$, both $\alpha$ and $\beta$ are nonempty.

An isotopy $\phi_{t}$ of $\mathbb{R}^{3}$ is called an $h$-isotopy if $\phi_{0}=i d$ and $\phi_{1}$ is a level-preserving map on $L$, i.e., $\rho \circ \phi_{i}=\rho$ on $L$. Note that $\phi_{t}$ does not have to be level-preserving on $L$ when $t \neq 0,1$. Since $\phi_{1}$ is level-preserving, the link $\phi_{1}(L)$ has the same width as $L$.

Lemma 2. Up to h-isotopy we may assume that $\alpha \subset Y_{-}$and $\beta \subset Y_{+}$.

Proof. By a level-preserving isotopy that shifts the whole link $L$ to the left we may assume that the 3-ball $B$ lies in $Y_{-}$. Let $I=[0, \epsilon]$ be an interval containing no critical value of $\rho$. So $L \cap Z_{I}$ can be assumed to be a set of vertical arcs from $\mathbb{R}^{2} \times 0$ to $\mathbb{R}^{2} \times \epsilon$. We may also assume that $B \cap Z_{I}=D^{\prime} \times I$. Let $f_{t}$ be an isotopy supported in $B$ that shrinks $\alpha$ into $D^{\prime} \times I$, i.e., $f_{0}=i d$ and $f_{1}(\alpha) \subset D^{\prime} \times I$. (Note that $f_{t}$ is not level-preserving.) There is now a level-preserving isotopy $g_{t}$ of $f_{1}(L)$, 
supported outside of $D^{\prime} \times I$, moving $\beta$ into $Y_{+}$. Let $h_{t}$ be the reverse isotopy of $f_{t}$, i.e., $h_{t}=f_{1-t}$. Then the union of these three isotopies is the required $h$-isotopy.

The separation of $\alpha$ and $\beta$ by the $(x, z)$-coordinate plane allows us to modify $\alpha$ by "vertical isotopy" without intersecting the rest of $L$. Let $\phi_{t}$ be an isotopy of the positive half of the $z$-axis. Then $i d \times \phi_{t}$ is an isotopy of $Y_{-}=\mathbb{R}_{-}^{2} \times \mathbb{R}_{+}$, which can be extended to an isotopy $f_{t}$ of $\mathbb{R}^{3}$ supported in a small neighborhood of $Y_{-}$in $\mathbb{R}_{+}^{3}$, and hence is the identity on $L-\beta$. This $f_{t}$ is called a vertical isotopy on $Y_{-}$ determined by $\phi_{t}$.

Denote by $m_{\alpha}$ the maximum value of $\rho(\alpha)$. Let $n_{\alpha}$ be the first local minimum level of $\alpha$, counted from the top down, and $n_{\alpha}=0$ if $\alpha$ has no local minimum. (It can be shown that $\alpha$ must have some local minima, but this is not necessary.) Define $m_{\beta}$ and $n_{\beta}$ similarly.

Lemma 3. Either $n_{\alpha}>m_{\beta}$ or $n_{\beta}>m_{\alpha}$.

Proof. Since $P$ is a thin level plane, at least one of $\alpha$ or $\beta$ contains some local minima; so $n_{\alpha}$ and $n_{\beta}$ cannot both be 0 . Without loss of generality we may assume that $n_{\alpha}<n_{\beta}$. Then above the level $n_{\beta}$ all the critical points of $L$ are local maxima. Note that lowering a local maximum of $\alpha$ through the level of a local maximum of $\beta$ will not change $w(L)$. If $m_{\alpha}>n_{\beta}$, then deforming a local maximum of $\alpha$ downward to a level just below $n_{\beta}$ would reduce $w(L)$, contradicting the minimality of $w(L)$.

By Lemma 3, we may assume without loss of generality that $m_{\alpha}<n_{\beta}$. In particular, $\beta$ must have some minima. Let $r \in\left(0, n_{\beta}\right)$ be such that $|P(r) \cap \beta|$ is minimal among all $|P(z) \cap \beta|, z \in\left[0, n_{\beta}\right]$. Let $I=[a, b]$ be a maximal interval in $[0, \infty)$ containing $r$ and having no critical value of $\beta$ in its interior. By the definition of $r$, one can see that $b$ is a local minimum level of $\beta$, and $a$ is either 0 or a local maximum level of $\beta$.

Let $f_{t}$ be a vertical isotopy supported in $Z_{[a, \infty)}$ that pushes $\alpha$ downward to $\alpha^{\prime}=f_{1}(\alpha)$ lying below the level $b$. Let $L^{\prime}$ be the presentation of $L$ obtained this way. We will show below that $w\left(L^{\prime}\right)<w(L)$, which then contradicts the assumption, completing the proof of Theorem 1 .

Since the isotopy $f_{t}$ is supported in $Z_{[a, \infty)}$, the critical points of $L$ below level $a$ and the widths of the corresponding level planes remain unchanged. So we need only calculate the sum of the widths for those level planes corresponding to critical points of $L$ and $L^{\prime}$ above level $a$. Denote by $P_{1}, \ldots, P_{k}$ (resp. $\left.P_{1}^{\prime}, \ldots, P_{k}^{\prime}\right)$ the level planes of $L$ corresponding to (i.e., lying just above) the critical points of $\alpha$ (resp. $\alpha^{\prime}$ ) above the level $a$, and by $R_{1}, \ldots, R_{h}$ those corresponding to critical points of $\beta$ above level $a$, labeled according to their height. Since $\beta$ has no critical values between $a$ and $b$, all $R_{j}$ are above level $b$. So we have

$$
\left|R_{j} \cap L\right|=\left|R_{j} \cap(\alpha \cup \beta)\right| \geq\left|R_{j} \cap \beta\right|=\left|R_{j} \cap L^{\prime}\right| .
$$

Also, since the top level of $\alpha$ is below $n_{\beta}$, by the choice of $r$ and the fact that $\left|P_{i} \cap \alpha\right|=\left|P_{i}^{\prime} \cap \alpha\right|$ we have

$$
\begin{aligned}
\left|P_{i} \cap(\alpha \cup \beta)\right| & =\left|P_{i} \cap \alpha\right|+\left|P_{i} \cap \beta\right| \geq\left|P_{i} \cap \alpha\right|+|P(r) \cap \beta| \\
& =\left|P_{i}^{\prime} \cap \alpha\right|+\left|P_{i}^{\prime} \cap \beta\right|=\left|P_{i}^{\prime} \cap L^{\prime}\right| .
\end{aligned}
$$

It follows that $w(L) \geq w\left(L^{\prime}\right)$, and equality holds if and only if it holds in all the above inequalities. On the other hand, since $P$ is a thinnest level of $L$, the level 
plane just below $b$ cannot be disjoint from $\alpha$, because otherwise it would be a thin level plane with width $|P(r) \cap \beta| \leq|P \cap \beta|<|P \cap L|$, contradicting the choice of $P$. Therefore, $\alpha$ must intersect the level plane $R_{1}$ lying just above level $b$. So $\left|R_{1} \cap L\right|>\left|R_{1} \cap L^{\prime}\right|$, and $w(L)>w\left(L^{\prime}\right)$, which contradicts the assumption that $L$ is in thin position.

Let $K_{1}$ and $K_{2}$ be knots in $S^{3}$. Putting them in thin position, with $K_{1}$ above $K_{2}$, then taking the obvious connected sum, we have a projection of $K=K_{1} \# K_{2}$ with width $w\left(K_{1}\right)+w\left(K_{2}\right)-2$. Hence we have $w(K) \leq w\left(K_{1}\right)+w\left(K_{2}\right)-2$. It was conjectured that $w(K)=w\left(K_{1}\right)+w\left(K_{2}\right)-2$ for all $K=K_{1} \# K_{2}$. See [RS, SS] for some work concerning this conjecture.

Recall that a knot $K$ is a small knot if its complement contains no closed essential surface. An essential surface $F$ in the knot exterior $E(K)=S^{3}-\operatorname{Int} N(K)$ is a meridional essential surface if $\partial F$ is a nonempty set of meridional curves on $\partial E(K)$. By CGLS, if $K$ is small, then its exterior contains no meridional essential planar surface. The following result is due to Rieck and Sedgwick [RS]. It proves the above conjecture for the connected sum of small knots $K_{1}$ and $K_{2}$.

Corollary 4 ([RS]). Let $K_{1}$ and $K_{2}$ be nontrivial knots in $S^{3}$ such that $E\left(K_{i}\right)=$ $S^{3}-\operatorname{Int} N\left(K_{i}\right)$ contains no meridional essential planar surfaces. Let $K$ be a thin position embedding of $K_{1} \# K_{2}$. Then there is a level sphere $S$ in $S^{3}$ intersecting $K$ in two points, decomposing $K$ into $K_{1}$ and $K_{2}$. In particular, $w\left(K_{1} \# K_{2}\right)=$ $w\left(K_{1}\right)+w\left(K_{2}\right)-2$.

Proof. Let $F$ be a sphere in $S^{3}$ that realizes the connected sum $K_{1} \# K_{2}$. Then $P=F \cap E(K)$ is a meridional essential planar surface. Since the exterior of $K_{i}$ contains no meridional essential planar surface, one can show that $P$ is the only meridional essential planar surface in $E(K)$.

It is easy to see that $K$ cannot be in bridge position: By Schubert's theorem $b(K)=b\left(K_{1}\right)+b\left(K_{2}\right)-1$. So a bridge position projection of $K$ can be obtained by putting $K_{i}$ in bridge position, with $K_{1}$ above $K_{2}$, taking the connected sum, then raising the maxima of $K_{2}$ over the minima of $K_{1}$. But the last operation would increase width; hence, if $K=K_{1} \# K_{2}$ is in bridge position, then it cannot be in thin position. (See $[\mathrm{RS}$ for an alternative proof.)

It now follows from Theorem 1 that if $K$ is in thin position, then some thinnest level surface $Q$ of $K$ is an essential planar surface in $S^{3}-\operatorname{Int} N(K)$. Since a meridional essential planar surface of $K$ is unique, $Q$ is the same as the surface $P$ above up to isotopy. Hence the result follows.

\section{ACKNOWLEDGMENT}

I would like to thank Abby Thompson for raising the question, and for some interesting conversations about the proof of Theorem 1. Thanks also go to Jennifer Schultens for some interesting discussions about thin position and width of the connected sum of knots.

\section{REFERENCES}

[CGLS] M. Culler, C. Gordon, J. Luecke and P. Shalen, Dehn surgery on knots, Ann. Math. 125 (1987), 237-300; 127 (1988), 663. MR 88a:57026] MR 89c:57015

[G] D. Gabai, Foliations and the topology of 3-manifolds, J. Differential Geom. 18 (1983), 445-503. MR 86a:57009 
[GL] C. McA. Gordon and J. Luecke, Knots are determined by their complements, J. Amer. Math. Soc. 2 (1989), 371-415. MR 90a:57006a

[HK] D. Heath and T. Kobayashi, Essential tangle decomposition from thin position of a link, Pacific J. Math. 179 (1997), 101-117. MR 98j:57007

[RS] Y. Rieck and E. Sedgwick, Thin position for a connected sum of small knots, Alg. Geom. Topology 2 (2002), 297-309. MR 2003d:57021

[SS] M. Scharlemann and J. Schultens, 3-manifolds with planar presentations and the width of satellite knots, preprint.

[ST] M. Scharlemann and A. Thompson, Heegaard splittings of (surface) $\times I$ are standard, Math. Ann. 295 (1993), 549-564. MR 94b:57020

[T1] Abigail Thompson, Thin position and the recognition problem for $S^{3}$, Math. Res. Lett. 1 (1994), 613-630. MR 95k:57015

[T2] Abigail Thompson, Thin position and bridge number for knots in the 3-sphere, Topology 36 (1997), 505-507. MR 97m:57013

[T3] Abigail Thompson, Lecture on a conference at UC Davis, 1996.

Department of Mathematics, University of Iowa, Iowa City, Iowa 52242

E-mail address: wu@math.uiowa.edu 